INPLASY

PROTOCOL

To cite: Jiménez-Lupion et al. Effect of muscle power training on fall risk in older adults: A systematic review and metaanalysis. Inplasy protocol 2021120073. doi:

10.37766/inplasy2021.12.0073

Received: 16 December 2021

Published: 16 December 2021

Corresponding author:

Daniel Jiménez Lupión

jlupion@ugr.es

Author Affiliation:

University of Granada

Support: Not aplicable.

Review Stage at time of this submission: Preliminary searches.

Conflicts of interest:

None declared.

\section{Effect of muscle power training on fall risk in older adults: A systematic review and meta-analysis}

Jiménez-Lupion, D1; Jerez-Mayorga, D2; Chirosa-Ríos, LJ3; Martínez-García, D4.

Review question / Objective: P: Older adults; I: Power training; C: Other type of exercise program; O: Fall Risk. Objective: To describe the effectiveness of muscle power training on fall risk in older adults.

Condition being studied: Healthy older adults or those with different pathologies who undergo a muscle power training program for the prevention of falls.

Eligibility criteria: Inclusion Criteria: Randomized Controlled Trial (RCT); Adults over 60 years of age, living independently in the community without disabilities and other diseases that make them unsuitable for exercise interventions; muscle power training of the lower limbs, without combining it with other types of exercise; Outcome: Fall Risk. Exclusion Criteria: Studies that used ergogenic drugs or aids; studies that manipulated diet; conference presentations, theses, books, editorials, review articles, and expert opinions; missing full text or incomplete data on outcome indicators.

INPLASY registration number: This protocol was registered with the International Platform of Registered Systematic Review and Meta-Analysis Protocols (INPLASY) on 16 December 2021 and was last updated on 16 December 2021 (registration number INPLASY2021120073).

\section{INTRODUCTION}

Review question / Objective: P: Older adults; I: Power training; C: Other type of exercise program; O: Fall Risk. Objective: To describe the effectiveness of muscle power training on fall risk in older adults.

Condition being studied: Healthy older adults or those with different pathologies who undergo a muscle power training program for the prevention of falls.

\section{METHODS}

Search strategy: The databases used were PubMed, Web of Science, Scopus and Sport Discus. The initial search was performed from October 2021 to November 2021, the following keywords were included: "Muscle power", "power training", "muscle power training", "high velocity resistance training", "high-speed resistance training", "high-speed power training", "fast-velocity resistance 
training", "fall", "falls", "fall risk", "risk of falls", "falling", "falls incidence", "older adults", "older people", "older persons", "older fallers", "elderly", "elderly people" and "elderly fallers".

Participant or population: Older adults (>60 years old).

Intervention: Power Training.

Comparator: Other type of exercise program.

Study designs to be included: Randomized Controlled Trial (RCT).

Eligibility criteria: Inclusion Criteria: Randomized Controlled Trial (RCT); Adults over 60 years of age, living independently in the community without disabilities and other diseases that make them unsuitable for exercise interventions; muscle power training of the lower limbs, without combining it with other types of exercise; Outcome: Fall Risk. Exclusion Criteria: Studies that used ergogenic drugs or aids; studies that manipulated diet; conference presentations, theses, books, editorials, review articles, and expert opinions; missing full text or incomplete data on outcome indicators.

Information sources: Electronic data bases (PubMed, Web of Science, Scopus and Sport Discus) and contact with authors.

Main outcome(s): The main outcome of this review is the risk of falling in older adults assessed by functional tests and questionnaires.

Quality assessment / Risk of bias analysis: The quality of evidence of the articles included in this review was assessed using the PEDro scale. This scale is based on criteria that allow us to identify whether the RCTs have sufficient internal validity and statistical information to interpret the results (external validity (item 1), internal validity (items 2-9), and statistical reporting (items 10-11). Each item is classified as yes or no ( 1 or 0 ) according to whether the criterion is clearly met in the study. The total score considers items two to 11 ; hence the maximum score is 10 (Cashin \& McAuley, 2020). Two independent researchers (DJ-L and DJ-M) evaluated the articles using this scale. In case of discrepancy, a third evaluator (DM-G) was consulted. After this evaluation, the assessment of those available articles in the PEDro database (https:// search.pedro.org.au/search) was consulted. Concerning the quality of evidence, it has been suggested that scores $<4$ are considered poor quality, 4 - 5 moderate quality, 6 - 8 good, and 9 - 10 excellent (Cashin \& McAuley, 2020).

Strategy of data synthesis: Data extraction was performed by two independent researchers (DJ-L and $D J-M)$; the information extracted was related to article identification (authors, country and year of publication), participant characteristics (sample, gender, and age), muscle power training protocols (exercises/sessions/ week) and the evaluation of the risk of falling. In addition to variables under study, main results, and missing data.

Subgroup analysis: We will conduct an analysis through subgroups according to the health conditions presented by the participants.

Sensitivity analysis: A sensitivity analysis was conducted to identify the presence of highly influential studies that might have biased the analysis.

Language: English.

Country(ies) involved: Spain.

Keywords: Older adults, Elderly, fall risk, muscle power.

Contributions of each author:

Author 1 - Daniel Jiménez-Lupión.

Email: jlupion@ugr.es

Author 2 - Daniel Jerez-Mayorga.

Author 3 - Luis Javier Chirosa-Ríos.

Author 4 - Darío Martínez-García. 